\title{
Prognostic Significance of Neutrophil-to-Lymphocyte Ratio in Patients with Sepsis: A Prospective Observational Study
}

\author{
Xuan Liu, Yong Shen, Hairong Wang, Qinmin Ge, Aihua Fei, and Shuming Pan \\ Department of Emergency, Xinhua Hospital, Shanghai Jiaotong University School of Medicine, 1665 Kongjiang Road, \\ Shanghai 200092, China \\ Correspondence should be addressed to Shuming Pan; drshumingpan@hotmail.com
}

Received 19 January 2016; Revised 3 March 2016; Accepted 9 March 2016

Academic Editor: Antonio Macciò

Copyright (C) 2016 Xuan Liu et al. This is an open access article distributed under the Creative Commons Attribution License, which permits unrestricted use, distribution, and reproduction in any medium, provided the original work is properly cited.

Background. The neutrophil-to-lymphocyte ratio (NLR) is an easily accessible biological marker that has been reported to represent disease severity. The aim of this study is to investigate the association between NLR and mortality in patients with sepsis. Methods. A total of 333 consecutive adult patients with sepsis were screened for eligibility in this prospective, observational study cohort. Severity scores and leukocyte counts were prospectively recorded upon entry to the intensive care unit (ICU). Receiver operating characteristic (ROC) curves and binary logistic regression models were used to assess the performance of NLR in predicting unfavorable outcome. Correlations between variables and disease severity were analyzed through Spearman correlation tests. Results. Median NLR levels were significantly higher in patients who died than in survivors. NLR had a modest power for predicting poor outcome as suggested by area under the curve (AUC) of $0.695 \pm 0.036$. Multivariate linear regression indicated that increased NLR levels were related to unfavorable outcome independently of the effect of possible confounders. Spearman correlation tests showed that there was a positive correlation between NLR levels and disease severity. Conclusions. Increased NLR levels were independently associated with unfavorable clinical prognosis in patients with sepsis. Further investigation is required to increase understanding of the pathophysiology of this relationship.

\section{Introduction}

Sepsis is a complicated condition and still a big challenge to both the developed and developing world. The reported morbidity of sepsis is constantly increasing, with severe sepsis and septic shock remaining among the major causes of death worldwide [1]. Although the mortality has been on the decline in recent years [2], low awareness, late identification, and improper management are still common [3]. Studies have found that one of the fundamental principles for the appropriate management of sepsis is early and accurate detection of the patients at high risk for death [4]. This is generally dependent on the application of scoring systems. Although various clinical biomarkers are widely explored [58], only a few have been currently applied in the clinical practice. Therefore, the search continues for preferable infection markers that may facilitate the prognosis prediction of sepsis.

The neutrophil-to-lymphocyte ratio (NLR), as a readily accessible biomarker, can be calculated based on a complete blood count. Although a growing body of evidence has shown that NLR is proposed as an independent predictor of poor survival in various clinical circumstances ranging from oncological patients $[9,10]$ to patients with cardiovascular diseases [11], there is no consensus about the relationship between NLR levels and clinical prognosis in patients with sepsis until now. In the context of infection, researchers in a recent study showed a reversed NLR evolution according to the timing of death [12], whereas some other studies suggested that NLR was not associated with mortality in patients with sepsis [13]. Consequently, the clinical usefulness of NLR in patients with sepsis is therefore still a matter of ongoing controversy and this question deserves further investigation.

In this prospective observational study, we sought to evaluate the potential association of NLR on intensive care unit (ICU) admission with the clinical prognosis in a consecutive series of adult patients with sepsis. 


\section{Material and Methods}

2.1. Study Design. This prospective trial recruited consecutive adult patients with sepsis admitted to the ICU of the Department of Emergency, Xinhua Hospital, Shanghai Jiaotong University School of Medicine, from October 2013 to October 2015.

For each patient with suspected infection, a complete diagnostic work-up was performed. The work-up comprised demographic and clinical characteristics, conventional risk factors, and important laboratory data including leukocyte counts, blood biochemistry, blood cultures, urine cultures, chest X-ray, and chest or abdominal computed tomography if necessary. Broad spectrum antimicrobial therapy was administered within 1 hour from the recognition of the septic status, always after collecting samples for microbiological culturing.

The inclusion criteria were as follows in the study: (1) age of at least 18 years; (2) sepsis due to one of the following infections: community acquired pneumonia, hospital acquired pneumonia, ventilator-associated pneumonia, acute pyelonephritis, intra-abdominal infection, or primary bacteremia; and (3) blood sampling within 24 hours from the presentation of signs of sepsis. We formulated a priori criterion to exclude patients according to the following criteria: (1) missing neutrophil and lymphocyte data on ICU admission; (2) missing covariate data for multivariable adjustments; (3) patients with immunosuppressive diseases mainly including cancer and HIV infection or patients with receiving immunosuppressive therapy; and (4) patients who were already in ICU for many days and became septic secondary. Patients were eligible for the final study cohort if they met the inclusion criteria and none of the exclusion criteria.

All the eligible patients were further classified according to standard definitions of sepsis, severe sepsis, and septic shock [14]. Specifically, sepsis was defined as the presence of infection together with systemic manifestations of infection; severe sepsis was defined as sepsis with sepsis-induced organ dysfunction or tissue hypoperfusion; septic shock was defined as sepsis-induced hypotension persisting despite adequate fluid resuscitation.

The study was approved by Shanghai Jiaotong University Xinhua Hospital Ethics Committee and was carried out in accordance with the Declaration of Helsinki. All patients were informed about the study and consented to participate. If the patient was unable to be informed, the next of kin was informed and provided consent for the patient to participate.

2.2. Blood Measurements. Venous blood ( $3 \mathrm{~mL})$ was collected from patients presenting to the ICU. The blood was drawn into an EDTA-containing tube (BD Vacutainer, Plymouth, UK) and centrifuged at 3,000 rpm for $15 \mathrm{~min}$, and plasma was frozen at $-80^{\circ} \mathrm{C}$ until analysis. Complete blood count was determined using the Beckman Coulter LH-750 Hematology Analyzer (Beckman Coulter, Inc., Fullerton, California). NLR was calculated as a ratio of circulating neutrophil and lymphocyte counts. The normal ranges for the leukocyte in our laboratory are 1.4-6.5 $\times 10^{9} / \mathrm{L}$ for neutrophil count and $1.2-3.4 \times 10^{9} / \mathrm{L}$ for lymphocyte count.
2.3. Disease Severity and Outcome. To evaluate the severity of sepsis upon presentation, the validated Acute Physiology and Chronic Health Evaluation II (APACHE II) score was calculated in all enrolled patients on admission. This score ranges from 0 to 71, with higher scores indicating more severe disease.

Furthermore, patients who survived and discharged from hospital were further followed up by telephone calls. The primary outcome of the study was defined as death from any cause within 28 days after admission to the ICU.

2.4. Statistical Analysis. Continuous variables were reported as mean values \pm standard deviation (SD) or median with interquartile range (IQR), while categorical variables were expressed as count and percentage. The statistical significance of intergroup differences was compared through unpaired Student's $t$-test or Mann-Whitney $U$ test for continuous variables and through Pearson's $\chi^{2}$ test for categorical variables. The ability of the variables to discriminate survivors from nonsurvivors was determined using receiver operating characteristic (ROC) curves. ROC curves showed sensitivity versus 1 - specificity such that area under the curve (AUC) varied from 0.5 to 1.0 , with increased values demonstrating higher discriminatory ability. Univariate logistic regression analyses were performed to separately examine the association between unfavorable outcome and each of the indicators. We also conducted forward stepwise multivariate logistic regression models to determine the independent predictors adjusted for the previously specified baseline covariates. Criteria of $P<0.05$ for entry and $P \geq 0.10$ for removal were imposed in this procedure. Correlations between variables and APACHE II score were analyzed through Spearman correlation tests. Two-sided $P$ value $<0.05$ was considered to represent a statistically significant difference. All analyses were performed by the IBM SPSS Statistics software version 19.0 (SPSS, Chicago, Illinois, USA).

\section{Results}

3.1. Baseline Characteristics of the Study Population. During the study period, there were 333 consecutive patients $(56.46 \%$ male; mean age, $70.26 \pm 15.79$ years) with complete neutrophil and lymphocyte data available, and all of these patients had complete data available for the primary outcome. A total of 253 patients survived and 80 died within 28 days after admission. The baseline clinical and laboratory characteristics of the patients are elaborated in Table 1. The median APACHE II score was 11 (IQR, 6 to 19). 137 patients (41.14\%) had sepsis, 149 patients $(44.74 \%)$ had severe sepsis, and the remaining 47 patients (14.11\%) had septic shock. 42 patients $(12.61 \%)$ received mechanical ventilation treatment, and 24 patients (7.21\%) received renal-replacement therapy. The commonest locations of infection were lung and abdomen, and the distribution of locations was similar among survivors and nonsurvivors. There was not any difference in NLR levels between groups with sepsis of pulmonary versus abdominal origin. The commonest isolated pathogens from the study cohort were Gram-negative microorganisms with a predominance of Escherichia coli, and blood cultures were positive 
TABLE 1: Baseline clinical and laboratory characteristics of the study subjects.

\begin{tabular}{|c|c|c|c|c|}
\hline \multirow{2}{*}{ Characteristics } & \multicolumn{4}{|c|}{ Patient group } \\
\hline & All patients & Survivors & Nonsurvivors & $P$ value \\
\hline \multicolumn{5}{|c|}{ Demographics and underlying conditions } \\
\hline Number of patients & 333 & 253 & 80 & - \\
\hline Males, number (\%) & $188(56.46 \%)$ & $137(54.15 \%)$ & $51(63.75 \%)$ & 0.131 \\
\hline Age (years) & $70.26 \pm 15.79$ & $67.82 \pm 16.59$ & $77.98 \pm 9.49$ & $0.000^{* *}$ \\
\hline COPD, number $(\%)$ & $38(11.41 \%)$ & $23(9.09 \%)$ & $15(18.75 \%)$ & $0.017^{*}$ \\
\hline Hypertension, number (\%) & $157(47.15 \%)$ & $115(45.45 \%)$ & $42(52.50 \%)$ & 0.271 \\
\hline CHD, number (\%) & $84(25.23 \%)$ & $55(21.74 \%)$ & $29(36.25 \%)$ & $0.008^{* *}$ \\
\hline Diabetes mellitus, number (\%) & $105(31.53 \%)$ & $80(31.62 \%)$ & $25(31.25 \%)$ & 0.950 \\
\hline Disease severity, number (\%) & & & & $0.008^{* *}$ \\
\hline Sepsis & $137(41.14 \%)$ & $117(46.25 \%)$ & $20(25.00 \%)$ & - \\
\hline Severe sepsis & $149(44.74 \%)$ & $107(42.29 \%)$ & $42(52.50 \%)$ & - \\
\hline Septic shock & $47(14.11 \%)$ & $29(11.46 \%)$ & $18(22.50 \%)$ & - \\
\hline \multicolumn{5}{|l|}{ Baseline parameters } \\
\hline APACHE II score & $11(6-19)$ & $9(6-14.5)$ & $20(14-29)$ & $0.000^{* *}$ \\
\hline WBC count $\left(10^{9} / \mathrm{L}\right)$ & $16.07 \pm 6.63$ & $15.35 \pm 6.11$ & $18.40 \pm 7.67$ & $0.000^{* *}$ \\
\hline Neutrophil $\left(10^{9} / \mathrm{L}\right)$ & $13.00(9.80-17.55)$ & $12.30(9.53-16.76)$ & $16.35(11.16-20.14)$ & $0.001^{* *}$ \\
\hline Lymphocyte $\left(10^{9} / \mathrm{L}\right)$ & $0.77(0.51-1.30)$ & $0.84(0.54-1.40)$ & $0.61(0.35-0.87)$ & $0.002^{* *}$ \\
\hline NLR & 17.85 (9.61-28.19) & $15.03(8.94-24.67)$ & $25.49(16.64-47.15)$ & $0.000^{* *}$ \\
\hline Platelet $\left(10^{9} / \mathrm{L}\right)$ & $191.24 \pm 68.57$ & $188.53 \pm 61.15$ & $199.96 \pm 79.88$ & 0.475 \\
\hline $\mathrm{RBC}$ count $\left(10^{9} / \mathrm{L}\right)$ & $4.10 \pm 0.83$ & $4.21 \pm 0.79$ & $3.74 \pm 0.84$ & 0.534 \\
\hline Hematocrit (\%) & $36.58 \pm 6.59$ & $37.34 \pm 6.12$ & $34.13 \pm 7.43$ & 0.066 \\
\hline RDW (\%) & $13.69 \pm 1.87$ & $13.40 \pm 1.36$ & $14.60 \pm 2.79$ & $0.000^{* *}$ \\
\hline Hemoglobin $(\mathrm{g} / \mathrm{L})$ & $123.11 \pm 22.99$ & $126.35 \pm 21.69$ & $112.75 \pm 24.13$ & 0.290 \\
\hline PCT $(n g / m L)$ & $18.54 \pm 7.28$ & $17.89 \pm 7.47$ & $20.60 \pm 7.13$ & 0.521 \\
\hline $\mathrm{CRP}(\mathrm{mg} / \mathrm{L})$ & $100.05 \pm 46.10$ & $97.45 \pm 47.43$ & $108.26 \pm 45.70$ & 0.140 \\
\hline $\mathrm{BNP}(\mathrm{ng} / \mathrm{mL})$ & $173.0(87.5-401.5)$ & $160.0(79.5-327.0)$ & $181.0(92.0-505.0)$ & $0.020^{*}$ \\
\hline Myoglobin (ng/mL) & $83.8(31.6-309.6)$ & $71.4(29.3-256.6)$ & $153.0(48.1-671.1)$ & $0.011^{*}$ \\
\hline CK-MB (ng/mL) & $2.7(1.4-6.0)$ & $2.5(1.2-5.0)$ & $3.2(2.1-9.0)$ & 0.348 \\
\hline Troponin $\mathrm{T}(\mathrm{ng} / \mathrm{mL})$ & $0.03(0.01-0.10)$ & $0.02(0.01-0.07)$ & $0.08(0.02-0.34)$ & $0.009^{* *}$ \\
\hline BUN (mmol/L) & $7.65(5.13-11.70)$ & $6.80(4.64-10.30)$ & $10.93(7.70-19.20)$ & 0.076 \\
\hline $\operatorname{Scr}(\mu \mathrm{mol} / \mathrm{L})$ & $121.54 \pm 50.63$ & $109.45 \pm 46.85$ & $160.26 \pm 67.97$ & $0.004^{* *}$ \\
\hline Cystatin C (mg/L) & $0.92(0.00-1.51)$ & $0.79(0.00-1.32)$ & $1.69(1.02-2.74)$ & $0.002^{* *}$ \\
\hline $\operatorname{ALT}(\mathrm{U} / \mathrm{L})$ & $29.0(19.0-49.8)$ & $28.0(18.0-56.0)$ & $30.0(19.0-48.5)$ & 0.075 \\
\hline AST (U/L) & $36.0(25.0-67.0)$ & $35.0(25.0-61.0)$ & $46.0(23.0-76.0)$ & 0.056 \\
\hline Bilirubin (mg/dL) & $22.61 \pm 11.43$ & $22.11 \pm 10.92$ & $24.19 \pm 13.07$ & 0.453 \\
\hline Albumin $(\mathrm{g} / \mathrm{L})$ & $33.25 \pm 6.04$ & $34.25 \pm 5.60$ & $30.04 \pm 6.32$ & $0.000^{* *}$ \\
\hline $\mathrm{FBG}(\mathrm{mmol} / \mathrm{L})$ & $8.37 \pm 5.08$ & $8.40 \pm 4.86$ & $8.26 \pm 5.12$ & 0.830 \\
\hline Lactic acid $(\mathrm{mmol} / \mathrm{L})$ & $2.0(1.2-2.8)$ & $1.8(1.0-2.4)$ & $2.3(1.6-3.4)$ & $0.002^{* *}$ \\
\hline \multicolumn{5}{|l|}{ Inflammatory cytokine } \\
\hline IL-1B (pg/mL) & $5.00(5.00-5.12)$ & $5.00(5.00-5.00)$ & $5.00(5.00-6.04)$ & 0.533 \\
\hline IL-2 receptor $(\mathrm{U} / \mathrm{mL})$ & $1222(801-1921)$ & $1142(764-1711)$ & $1574(1070-3379)$ & $0.000^{* *}$ \\
\hline IL-6 (pg/mL) & $27.9(14.0-68.4)$ & $26.1(12.3-58.3)$ & $49.8(20.0-133.0)$ & 0.052 \\
\hline IL-8 (pg/mL) & $53.9(21.3-153.5)$ & $44.7(18.7-139.0)$ & $106.5(35.1-242.8)$ & 0.289 \\
\hline IL-10 (pg/mL) & $5.9(5.0-12.6)$ & $5.7(5.0-8.9)$ & $9.0(5.0-26.7)$ & 0.099 \\
\hline TNF- $\alpha(\mathrm{pg} / \mathrm{mL})$ & $22.6(15.9-34.4)$ & $22.2(15.6-33.7)$ & $26.8(16.6-41.2)$ & 0.066 \\
\hline CD64 & $3.6(1.6-6.2)$ & $2.9(1.5-5.8)$ & $3.9(1.7-6.3)$ & $0.034^{*}$ \\
\hline Site of infection, number (\%) & & & & 0.602 \\
\hline Lung & $184(55.26 \%)$ & $135(53.36 \%)$ & $49(61.25 \%)$ & - \\
\hline Abdomen & $70(21.02 \%)$ & $56(22.13 \%)$ & $14(17.50 \%)$ & - \\
\hline Urinary tract & $55(16.52 \%)$ & $44(17.39 \%)$ & $11(13.75 \%)$ & - \\
\hline Other & $24(7.21 \%)$ & $18(7.11 \%)$ & $6(7.50 \%)$ & - \\
\hline
\end{tabular}


TABLE 1: Continued.

\begin{tabular}{lccc}
\hline Characteristics & \multicolumn{2}{c}{ Patient group } & Nonsurvivors \\
\hline Intervention, number (\%) & All patients & Survivors & $25(31.25 \%)$ \\
$\quad$ Mechanical ventilation & $42(12.61 \%)$ & $17(6.72 \%)$ & $14(17.50 \%)$ \\
$\quad$ Renal-replacement therapy & $24(7.21 \%)$ & $10(3.95 \%)$ & \\
Length of stay & & & $6(2-12)$ \\
In the ICU (days) & $4.5(2-9)$ & $4(1-8)$ & $11(7-16)$ \\
In the hospital (days) & $10(7-14)$ & $9(7-13)$ & $0.000^{* *}$ \\
\hline
\end{tabular}

COPD: chronic obstructive pulmonary disorder; CHD: coronary heart disease; APACHE II: Acute Physiology and Chronic Health Evaluation II; WBC: white blood cell; NLR: neutrophil-to-lymphocyte ratio; RBC: red blood cell; RDW: red blood cell distribution width; PCT: procalcitonin; CRP: C-reactive protein; BNP: brain natriuretic peptide; CK-MB: creatine kinase-MB; BUN: blood urea nitrogen; Scr: serum creatinine; ALT: alanine transaminase; AST: aspartate transaminase; FBG: fasting blood glucose; IL: interleukin; TNF- $\alpha$ : tumor necrosis factor- $\alpha$.

Data are expressed as number (\%), mean (standard deviation, $\mathrm{SD}$ ), or median (interquartile range, IQR) as appropriate.

Significant differences are marked by ${ }^{*}(P<0.05)$ or ${ }^{* *}(P<0.01)$.

in $33.63 \%$ of all patients. There were 67 bacteremic patients among the survivors and 45 bacteremic patients among the nonsurvivors. NLR levels of the patients with positive blood culture were significantly higher than the ones with negative blood culture (22.65 (IQR, 12.60 to 36.93 ) versus 14.66 (8.15 to 25.62), $P=0.000$ ). Although the median length of stay in the hospital was similar between survivors and nonsurvivors $(P=0.468)$, the median length of stay in the ICU was significantly longer in nonsurvivors $(P=$ 0.041 ). In addition, the proportion of nonsurvivors receiving mechanical ventilation or renal-replacement therapy was greater than survivors $(P=0.000)$.

The median NLR for the entire cohort was 17.85 (IQR, 9.61 to 28.19). The neutrophil count of nonsurvivors on admission was higher than that of survivors $(P=0.001)$. Of note, the lymphocyte count was much less $(P=0.002)$ with an increased NLR $(P=0.000)$ in the nonsurvivors compared to patients that survived. Nonsurvivors tended to be older and have higher baseline levels of APACHE II score, as well as more white blood cell (WBC) count compared with the survivors. However, there was no statistically significant difference in nonsurvivors versus survivors with respect to other conventional infection markers including procalcitonin $(\mathrm{PCT})(P=0.521)$ and $\mathrm{C}$-reactive protein $(\mathrm{CRP})(P=0.140)$.

3.2. Value of Indicators in Predicting Unfavorable Outcome. ROC curves were constructed to evaluate the performance of indicators in differentiating nonsurvivors from survivors, and the AUC for each indicator was compared. The AUC, optimal cutoff value, sensitivity, and specificity of each indicator are presented in Table 2. NLR had a modest power for predicting unfavorable outcome as suggested by AUC of $0.695 \pm 0.036$, which was less than that of baseline APACHE II score (0.828 \pm $0.026)$ but greater than that of neutrophil $(0.633 \pm 0.036)$ and lymphocyte $(0.650 \pm 0.035)$. NLR $\geq 23.8$ was proposed as the optimal cutoff value, which provided a sensitivity of $81.3 \%$ and a specificity of $53.6 \%$ for predicting mortality in sepsis (Figure 1).

Furthermore, we performed univariate logistic regression analyses to examine the associations of each variable with unfavorable outcome and calculated the standardized regression coefficient $(\beta)$ and the odds ratio (OR) for each variable.

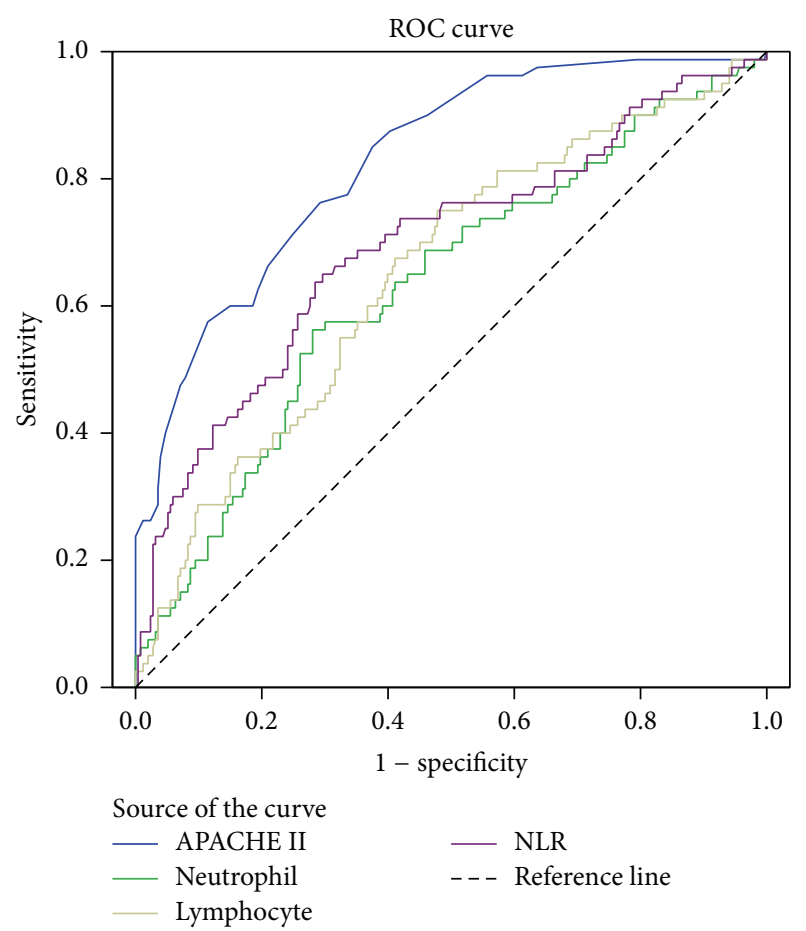

FIGURE 1: Receiver operating characteristic (ROC) curves for Acute Physiology and Chronic Health Evaluation II (APACHE II) score, neutrophil, lymphocyte, and neutrophil-to-lymphocyte ratio (NLR). NLR had a modest power for predicting unfavorable outcome as suggested by area under the curve (AUC) of $0.695 \pm 0.036, P=0.000$.

As shown in Table 3, baseline APACHE II score had the greatest absolute value of standardized $\beta$ value $(0.2342)$. The absolute value of standardized $\beta$ value for NLR was 0.0378 and the unadjusted OR was 1.038 (95\% confidence interval (CI), 1.008-1.070, $P=0.013$ ), indicating that NLR had a power for predicting unfavorable outcome.

3.3. Independent Prognosis Significance of NLR. We conducted a forward stepwise multivariate logistic regression model to determine the independent predictors of adverse outcome. The results are shown in Table 4. Baseline APACHE 
TABLE 2: Performance of variables in predicting unfavorable outcome.

\begin{tabular}{|c|c|c|c|c|c|}
\hline Variables & AUC ROC & $P$ value & Cutoff value & Sensitivity (\%) & Specificity (\%) \\
\hline APACHE II score & $0.828 \pm 0.026$ & $0.000^{* *}$ & $\geq 16.5$ & 76.3 & 70.8 \\
\hline Neutrophil & $0.633 \pm 0.036$ & $0.000^{* *}$ & $\geq 14.2$ & 73.8 & 45.8 \\
\hline Lymphocyte & $0.650 \pm 0.035$ & $0.000^{* *}$ & $\leq 0.64$ & 75.0 & 58.1 \\
\hline NLR & $0.695 \pm 0.036$ & $0.000^{* *}$ & $\geq 23.8$ & 81.3 & 53.6 \\
\hline
\end{tabular}

AUC ROC: area under the receiver operating characteristic curve; APACHE II: Acute Physiology and Chronic Health Evaluation II; and NLR: neutrophil-tolymphocyte ratio.

Significant differences are marked by ${ }^{* *}(P<0.01)$.

TABLE 3: Univariate odds ratios of variables for predicting unfavorable outcome.

\begin{tabular}{|c|c|c|c|c|}
\hline Variables & Standard $\beta$ value & OR & $95 \% \mathrm{CI}$ & $P$ value \\
\hline APACHE II score & 0.2342 & 1.168 & $1.102-1.238$ & $0.000^{* *}$ \\
\hline Neutrophil & -0.0875 & 0.916 & $0.766-1.096$ & 0.339 \\
\hline Lymphocyte & 0.0671 & 1.069 & $0.808-1.416$ & 0.639 \\
\hline NLR & 0.0378 & 1.038 & $1.008-1.070$ & $0.013^{*}$ \\
\hline WBC & 0.0195 & 1.020 & $0.955-1.089$ & 0.558 \\
\hline Lactic acid & 0.0461 & 1.047 & $0.860-1.276$ & 0.647 \\
\hline Age & 0.0813 & 1.085 & $1.039-1.132$ & $0.000^{* *}$ \\
\hline
\end{tabular}

APACHE II: Acute Physiology and Chronic Health Evaluation II; NLR: neutrophil-to-lymphocyte ratio; and WBC: white blood cell.

The OR indicates the risk of obtaining unfavorable outcome. Standard $\beta$ value was calculated using the semistandardization method ( $X$ standardization).

Significant differences are marked by ${ }^{*}(P<0.05)$ or ${ }^{* *}(P<0.01)$.

TABLE 4: Independent predictors of unfavorable outcome by multivariate logistic regression analysis.

\begin{tabular}{lcccc}
\hline Variables & Standard $\beta$ value & OR & $95 \%$ CI & $P$ value \\
\hline APACHE II score & 0.2639 & 1.168 & $1.108-1.230$ & $0.000^{* *}$ \\
NLR & 0.0471 & 1.043 & $1.012-1.083$ & $0.016^{*}$ \\
Age & 0.0745 & 1.077 & $1.034-1.122$ & $0.000^{* *}$ \\
\hline
\end{tabular}

APACHE II: Acute Physiology and Chronic Health Evaluation II; NLR: neutrophil-to-lymphocyte ratio.

The OR indicates the risk of obtaining unfavorable outcome. Standard $\beta$ value was calculated using the semistandardization method ( $X$ standardization), Variables not listed in the table were removed from the stepwise analysis.

Significant differences are marked by ${ }^{*}(P<0.05)$ or ${ }^{* *}(P<0.01)$.

II score (adjusted OR, 1.168; 95\% CI, 1.108-1.230; and $P=$ 0.000 ) and NLR (adjusted OR, 1.043; 95\% CI, 1.012-1.083; and $P=0.016$ ) were the independent predictors which entered the final prediction model, indicating that higher NLR levels increased the risk of shifting to an unfavorable outcome independently of the effect of possible confounders. In addition, old age was also independently related to unfavorable outcome (adjusted OR, 1.077; 95\% CI, 1.034-1.122; and $P=0.000)$.

3.4. Association between NLR and Disease Severity. Patients with severe sepsis or septic shock tended to have higher baseline levels of APACHE II score, neutrophil count, and NLR, as well as lower lymphocyte count compared with patients with sepsis (Table 5). As APACHE II score increased, neutrophil count and NLR levels consistently increased, while lymphocyte count consistently decreased. Furthermore, the correlations between neutrophil count, lymphocyte count, NLR, and APACHE II score were analyzed through Spearman correlation tests. A positive correlation was reported between NLR levels and APACHE II score at baseline $(r=0.641$, $P=0.000)$, suggesting that NLR levels were positively proportional to disease severity. In addition, neutrophil count was positively correlated with disease severity $(r=0.383, P=$ 0.000 ), while lymphocyte count was inversely proportional to disease severity $(r=-0.474, P=0.000)$.

\section{Discussion}

In the current prospective study, we further explored the prognosis significance of the NLR in patients with sepsis and found that the NLR measured at the time of admission to ICU was associated with 28-day mortality and correlated well with disease severity, according to APACHE II score. NLR was able to accurately stratify patients in terms of short-term mortality. These findings remained robust after adjusting for several potential covariates, suggesting that increased NLR was independently associated with unfavorable outcome in patients with sepsis. In our opinion, the strength of the NLR is the possibility of implementing this parameter simply by using already available biomarkers (neutrophil count and lymphocyte count). Therefore, this ratio is easy to integrate in clinical practice and cost effective. 
TABLE 5: Correlations of indicators with disease severity.

\begin{tabular}{|c|c|c|c|c|}
\hline Variables & Sepsis & Severe sepsis & Septic shock & $P$ value \\
\hline APACHE II score & $8.00(5.00-11.50)$ & $13.00(9.00-19.00)$ & $21.50(14.75-30.25)$ & $0.000^{* *}$ \\
\hline Neutrophil & $11.75(9.52-16.65)$ & $13.35(10.44-18.22)$ & $15.98(10.14-21.29)$ & $0.016^{*}$ \\
\hline Lymphocyte & $1.12(0.71-1.70)$ & $0.67(0.45-0.95)$ & $0.51(0.26-0.78)$ & $0.000^{* *}$ \\
\hline NLR & $11.11(6.98-18.24)$ & $22.67(12.35-31.89)$ & $31.50(22.56-46.94)$ & $0.000^{* *}$ \\
\hline
\end{tabular}

APACHE II: Acute Physiology and Chronic Health Evaluation II; NLR: neutrophil-to-lymphocyte ratio. Significant differences are marked by ${ }^{*}(P<0.05)$ or ${ }^{* *}(P<0.01)$.

Although the available information is still far from sufficient to comprehend thoroughly the economic burden of sepsis on an international scale, current studies demonstrate that sepsis has been a serious public health problem $[15,16]$. The patients with septic shock have high risk of death, complications, and resource utilization [17]. Undoubtedly, the pivotal measure of improving outcome is to identify the septic patients with poor prognosis accurately [4]. Although recently introduced infection markers such as several cytokines and markers like soluble urokinase plasminogen activator receptor, endothelin-1, and copeptin have raised concerns in risk stratification and prognosis prediction, the application of these infection markers is still limited by validation, costs, and accessibility. To the best of our knowledge, immunocompetent leukocyte plays an important role in the systemic inflammatory response to infection. Most of the prognostic scores use leukocytosis (above $12.0 \times 10^{9} / \mathrm{L}$ ) or leukopenia (below $4.0 \times 10^{9} / \mathrm{L}$ ) as a severity index, but few consider the leukocyte subpopulations $[14,18]$. Significant differences exist between circulating neutrophil and lymphocyte counts and, consequently, their ratio-referred to as the NLR-has been increasingly used in the prediction of the severity or prognosis in different clinical settings, including systemic inflammation and sepsis [19-21], ischemic events [22], and cancer [23, 24].

The cause responsible for NLR elevations correlating with poor outcome in patients with sepsis remains unclear, although there are a variety of plausible explanations. One of the most convincing explanations is based primarily on the physiological link between neutrophilia and lymphopenia with systemic inflammation and stress. The evolution of these leukocyte subpopulations may differ based on their respective role in the inflammatory response. Initially, Zahorec [9] explored the use of NLR in septic ICU patients and suggested that NLR was proposed as an indicator of the patient's response to inflammatory insult. Increased numbers of neutrophil implied that nidus of infection was not eradicated, which further induced depression of lymphocyte. Another large-scale study further found the presence of persistent lymphopenia and neutrophilia in trauma patients and patients who met the criteria for the systemic inflammatory response syndrome [25]. Evidence is growing that neutrophil is the key cellular component of host defense in the innate immune system against infectious injury, while lymphocyte is considered as the major cellular line of the adaptive immune system. Lymphocyte plays a key role in the regulation of inflammatory response, and their loss due to continuous sepsis-induced apoptosis may lead to the immune system suppression and nonresolution of inflammation [25, 26]. Taken together, the sustainability of infection and the incomplete eradication of nidus of infection are responsible for the increase of neutrophils production by the medulla and decrease lymphocytes counts by apoptosis and others mechanisms. Therefore, the resulting increase in NLR may identify patients who are in a state of nonresolution of inflammation, along with concomitant decreased survival rates.

We assessed the association between NLR and outcome in patients with sepsis. Similar to the findings of a previous clinical trial [9], our study clearly showed that the risk of death was associated with neutrophil count increase, lymphocyte count decrease, and subsequent increase in the NLR in the patients with sepsis at the time of admission to the ICU. In contrast, Salciccioli et al. found that there was no statistically significant relationship between NLR and mortality in patients with sepsis [13]. Another recent research indicated that the NLR on admission was significantly lower in patients who died before day 5 of septic shock onset than in survivors, and an increased NLR from day 1 to day 5 was associated with late death [12]. Why the results of these studies were conflicting can be attributed to the following reasons. First, the definition of primary outcome in different studies is inconsistent. Some of them defined 28-day mortality as outcome; nevertheless, some others defined early (before day 5 of septic shock onset) or late (on or after day 5 of septic shock onset) ICU mortality as outcome. Second, the disease severity of enrolled patients differs in the different studies. Some researches included both patients with sepsis and those with severe sepsis or septic shock when the other researches just enrolled patients with septic shock.

This study has a number of limitations that should be taken into consideration. First, we undertook a single-center observational study, and, as with any observational study, the potential remains for residual confounding. Thus, the results should be validated in other settings. Second, for some patients, repeated measurement data were available on the first day. We always used the first one and thus missed some information related to intraday cell count variations. Third, we just analyzed circulating neutrophil and lymphocyte counts and did not explore the different subpopulations of lymphocytes. The further work in our laboratory is to compare the subpopulations of lymphocytes, but the research data have not been shown in this study at present. Fourth, recently developed infection markers like soluble urokinase plasminogen activator receptor, endothelin-1, and copeptin were not evaluated and compared with NLR in the study. 
Finally, our sample size was so limited that the results should be further confirmed in a larger scale.

\section{Conclusions}

The NLR was associated with 28-day mortality in patients with sepsis. Use of the NLR may better help the physician stratify patients into prognostic categories. The present study can be considered as a preliminary attempt to clarify the strengths of NLR in prognosis prediction. However, the mechanisms underlying the association are yet to be fully elucidated and should be the focus of future prospective clinical research.

\section{Abbreviations}

NLR: $\quad$ Neutrophil-to-lymphocyte ratio

ICU: Intensive care unit

APACHE II: Acute Physiology and Chronic Health

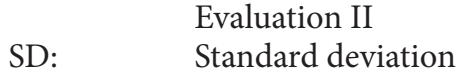

IQR: Interquartile range

ROC: $\quad$ Receiver operating characteristic

AUC: $\quad$ Area under the curve

WBC: White blood cell

PCT: $\quad$ Procalcitonin

CRP: $\quad$ C-reactive protein

OR: $\quad$ Odds ratio

CI: Confidence interval.

\section{Competing Interests}

The authors declare that there is no conflict of interests.

\section{Acknowledgments}

This work was financially supported by the grant from the 2013-2014 National Clinical Key Specialty Construction Project and the Health Bureau Scientific Research Foundation of Shanghai (GWDTR201219).

\section{References}

[1] The ARISE Investigators and the ANZICS Clinical Trials Group, "Goal-directed resuscitation for patients with early septic shock," The New England Journal of Medicine, vol. 371, no. 16, pp. 1496-1506, 2014.

[2] K.-M. Kaukonen, M. Bailey, S. Suzuki, D. Pilcher, and R. Bellomo, "Mortality related to severe sepsis and septic shock among critically ill patients in Australia and New Zealand, 2000-2012," The Journal of the American Medical Association, vol. 311, no. 13, pp. 1308-1316, 2014.

[3] K. Reinhart, R. Daniels, N. Kissoon, J. O’Brien, F. R. Machado, and E. Jimenez, "The burden of sepsis-a call to action in support of World Sepsis Day 2013," Journal of Critical Care, vol. 28, no. 4, pp. 526-528, 2013.

[4] R. P. Dellinger, M. M. Levy, A. Rhodes et al., "Surviving Sepsis Campaign: international guidelines for management of severe sepsis and septic shock, 2012," Intensive Care Medicine, vol. 39, no. 2, pp. 165-228, 2013.

[5] S. Vera, R. Martínez, J. G. Gormaz, A. Gajardo, F. Galleguillos, and R. Rodrigo, "Novel relationships between oxidative stress and angiogenesis-related factors in sepsis: new biomarkers and therapies," Annals of Medicine, vol. 47, no. 4, pp. 289-300, 2015.

[6] D. Y. Hong, J. W. Kim, J. H. Paik et al., "Value of plasma neutrophil gelatinase-associated lipocalin in predicting the mortality of patients with sepsis at the emergency department," Clinica Chimica Acta, vol. 452, pp. 177-181, 2016.

[7] A. Linder, R. Arnold, J. H. Boyd et al., "Heparin-binding protein measurement improves the prediction of severe infection with organ dysfunction in the emergency department," Critical Care Medicine, vol. 43, no. 11, pp. 2378-2386, 2015.

[8] M. Hur, H. Kim, H. J. Kim et al., "Soluble ST2 has a prognostic role in patients with suspected sepsis," Annals of Laboratory Medicine, vol. 35, no. 6, pp. 570-577, 2015.

[9] R. Zahorec, "Ratio of neutrophil to lymphocyte counts-rapid and simple parameter of systemic inflammation and stress in critically ill," Bratislavske Lekarske Listy, vol. 102, no. 1, pp. 5-14, 2001.

[10] M. H. Kang, S.-I. Go, H.-N. Song et al., "The prognostic impact of the neutrophil-to-lymphocyte ratio in patients with smallcell lung cancer," British Journal of Cancer, vol. 111, no. 3, pp. 452-460, 2014.

[11] B. Ayça, F. Akln, O. Celik et al., "Neutrophil to lymphocyte ratio is related to stent thrombosis and high mortality in patients with acute myocardial infarction," Angiology, vol. 66, no. 6, pp. 545552, 2015.

[12] F. Riché, E. Gayat, R. Barthélémy, M. Le Dorze, J. Matéo, and D. Payen, "Reversal of neutrophil-to-lymphocyte count ratio in early versus late death from septic shock," Critical Care, vol. 19, article 439, 2015.

[13] J. D. Salciccioli, D. C. Marshall, M. A. Pimentel et al., "The association between the neutrophil-to-lymphocyte ratio and mortality in critical illness: an observational cohort study," Critical Care, vol. 19, no. 1, article 13, 2015.

[14] M. M. Levy, M. P. Fink, J. C. Marshall et al., "2001 SCCM/ ESICM/ACCP/ATS/SIS international sepsis definitions conference," Intensive Care Medicine, vol. 29, no. 4, pp. 530-538, 2003.

[15] I. Jawad, I. Lukšić, and S. B. Rafnsson, "Assessing available information on the burden of sepsis: global estimates of incidence, prevalence and mortality," Journal of Global Health, vol. 2, no. 1, Article ID 010404, 2012.

[16] D. F. Gaieski, J. M. Edwards, M. J. Kallan, and B. G. Carr, "Benchmarking the incidence and mortality of severe sepsis in the United States," Critical Care Medicine, vol. 41, no. 5, pp. 11671174, 2013.

[17] D. A. Harrison, C. A. Welch, and J. M. Eddleston, "The epidemiology of severe sepsis in England, Wales and Northern Ireland, 1996 to 2004: secondary analysis of a high quality clinical database, the ICNARC Case Mix Programme Database," Critical Care, vol. 10, no. 2, article R42, 2006.

[18] J.-L. Vincent and R. Moreno, "Clinical review: scoring systems in the critically ill," Critical Care, vol. 14, no. 2, article 207, 2010.

[19] B. B. Sen, E. N. Rifaioglu, O. Ekiz et al., "Neutrophil to lymphocyte ratio as a measure of systemic inflammation in psoriasis," Journal of Cutaneous and Ocular Toxicology, vol. 33, no. 3, pp. 223-227, 2014.

[20] C. P. C. de Jager, P. T. L. van Wijk, R. B. Mathoera, J. de JonghLeuvenink, T. van der Poll, and P. C. Wever, "Lymphocytopenia 
and neutrophil-lymphocyte count ratio predict bacteremia better than conventional infection markers in an emergency care unit," Critical Care, vol. 14, no. 5, article R192, 2010.

[21] C. P. C. de Jager, P. C. Wever, E. F. A. Gemen et al., "The neutrophil-lymphocyte count ratio in patients with community-acquired pneumonia," PLOS ONE, vol. 7, no. 10, Article ID e46561, 2012.

[22] A. J. Grau, A. W. Boddy, D. A. Dukovic et al., "Leukocyte count as an independent predictor of recurrent ischemic events," Stroke, vol. 35, no. 5, pp. 1147-1152, 2004.

[23] T. Motomura, K. Shirabe, Y. Mano et al., "Neutrophillymphocyte ratio reflects hepatocellular carcinoma recurrence after liver transplantation via inflammatory microenvironment," Journal of Hepatology, vol. 58, no. 1, pp. 58-64, 2013.

[24] G. Malietzis, M. Giacometti, A. Askari et al., "A preoperative neutrophil to lymphocyte ratio of 3 predicts disease-free survival after curative elective colorectal cancer surgery," Annals of Surgery, vol. 260, no. 2, pp. 287-292, 2014.

[25] D. S. Heffernan, S. F. Monaghan, R. K. Thakkar, J. T. Machan, W. G. Cioffi, and A. Ayala, "Failure to normalize lymphopenia following trauma is associated with increased mortality, independent of the leukocytosis pattern," Critical Care, vol. 16, no. 1, article R12, 2012.

[26] T. Menges, J. Engel, I. Welters et al., "Changes in blood lymphocyte populations after multiple trauma: Association with posttraumatic complications," Critical Care Medicine, vol. 27, no. 4, pp. 733-740, 1999. 


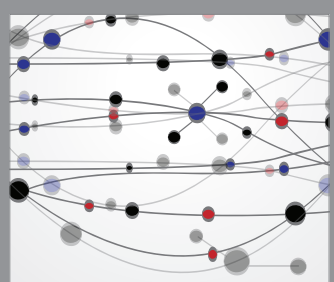

The Scientific World Journal
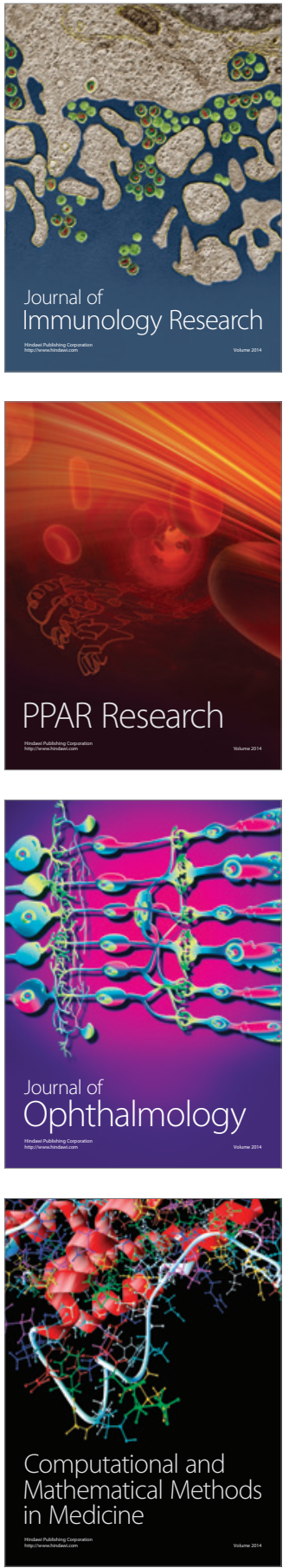

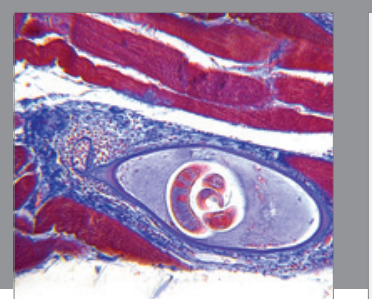

Gastroenterology Research and Practice

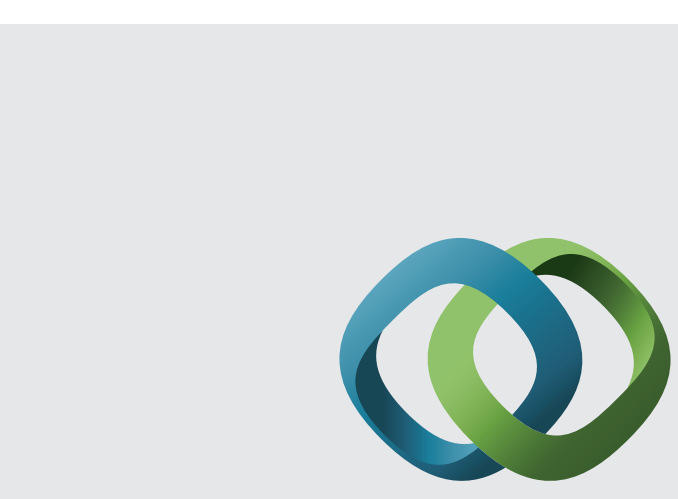

\section{Hindawi}

Submit your manuscripts at

http://www.hindawi.com
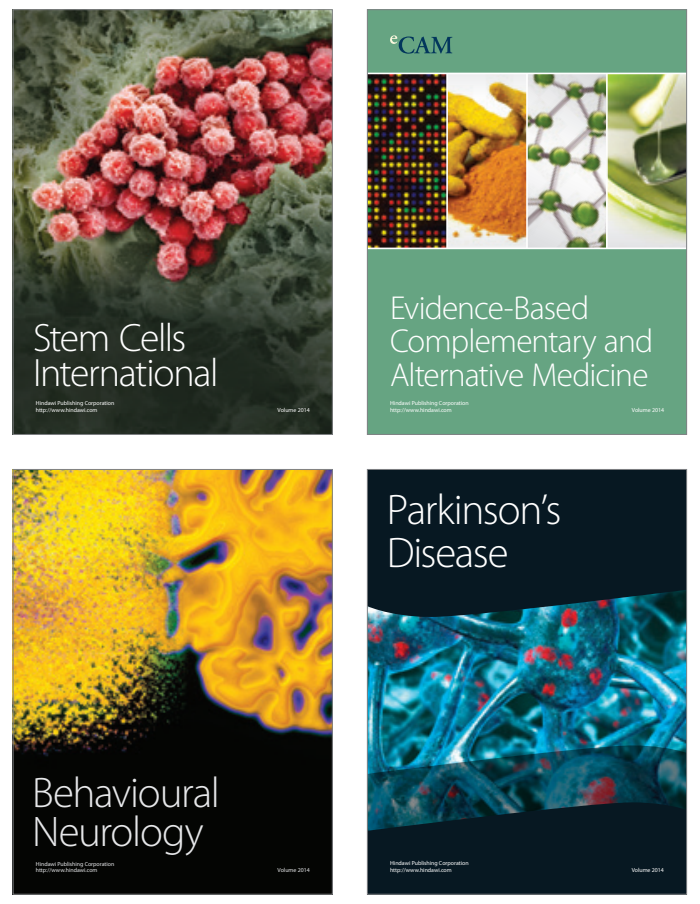
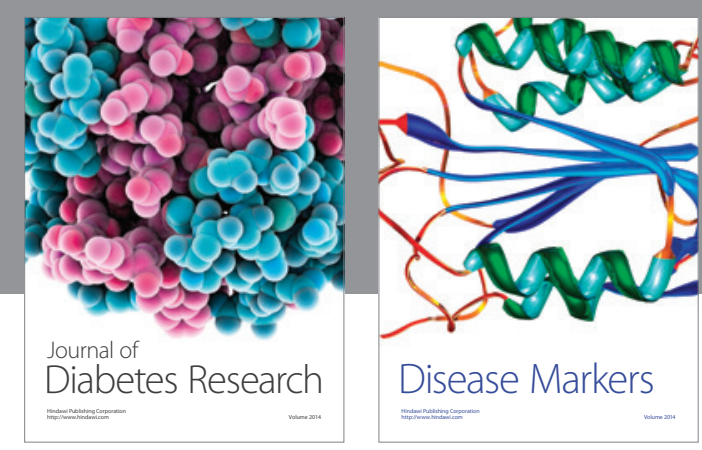

Disease Markers
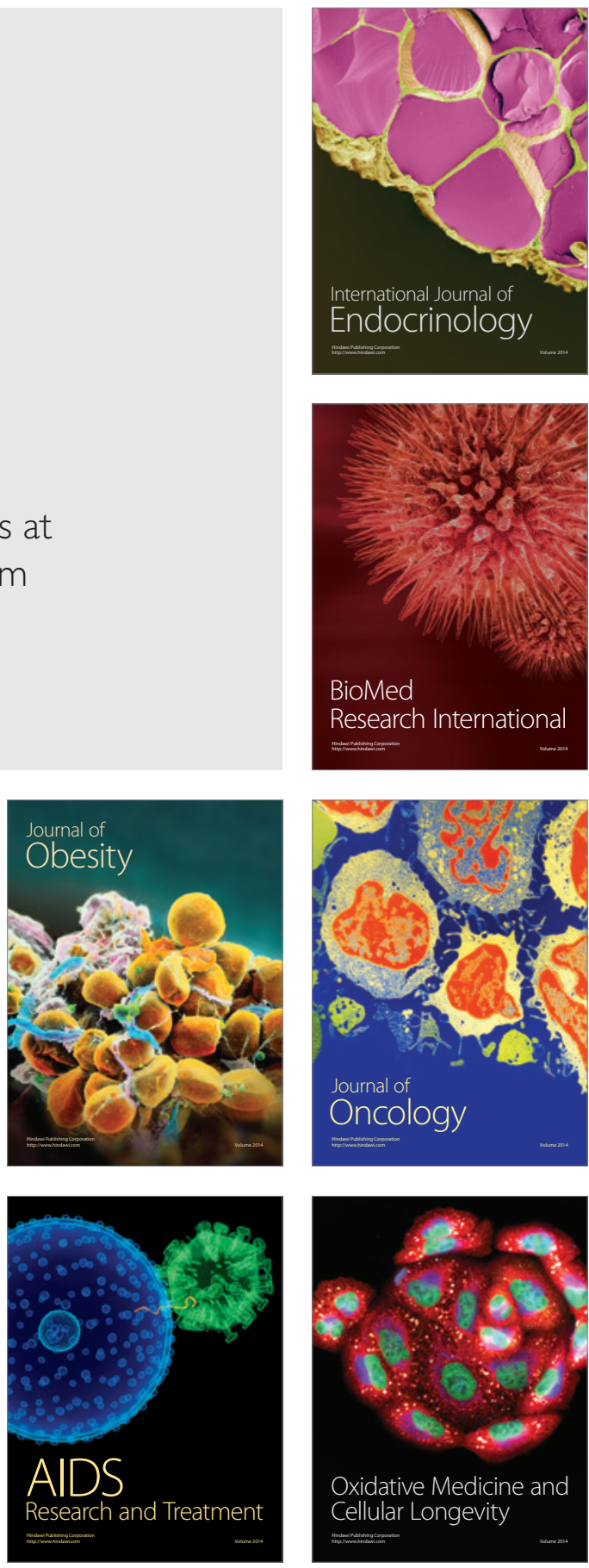